\title{
Correction to: Neuroprotective effect of naturally occurring RXR agonists isolated from Sophora tonkinensis Gagnep. on amyloid- $\beta$-induced cytotoxicity in PC1 2 cells
}

\author{
Wei Wang $^{1} \cdot$ Ken-ichi Nakashima $^{1} \cdot$ Takao Hirai $^{1} \cdot$ Makoto Inoue $^{1}$
}

Published online: 9 April 2019

(c) The Japanese Society of Pharmacognosy 2019

\section{Correction to: \\ Journal of Natural Medicines (2019) 73:154-162 \\ https://doi.org/10.1007/s11418-018-1257-z}

In the original publication of the article, Table 1 and Fig. 1 were incorrectly published.
Table 1 was not properly aligned. It also contained an error in the base sequence of the forward primer of Rat ABCA1. In Fig. 1, the structure formula of SPF1 was incorrect. The correct Table 1 and Fig. 1 are provided below:

Table 1 List of primers used in the present study

\begin{tabular}{lll}
\hline Gene & Forward primer & Reverse primer \\
\hline Rat ABCA1 & TGC GGC GTG AAG CCT GTC ATC T & ACG ACC AGT GTA GCA GGG ACC A \\
Rat $\beta$-actin & CCC ATC TAT GAG GGT TAC GC & TTT AAT GTC ACG CAC GAT TTC \\
Human SREBC-1c & TCA GCG AGG CGG CTT TGG AGC AG & CAT GTC TTC GAT GTC GGT CAG \\
Human GAPDH & CGA GAT CCC TCC AAA ATC AA & GGT GCT AAG CAG TTG GTG GT \\
\hline
\end{tabular}

The original article can be found online at https://doi.org/10.1007/ s11418-018-1257-z.

Makoto Inoue

minoue@dpc.aichi-gakuin.ac.jp

Wei Wang

wangwei880726@gmail.com

Ken-ichi Nakashima

nakasima@dpc.agu.ac.jp

Takao Hirai

t-hirai@dpc.agu.ac.jp

1 Laboratory of Natural Resources, School of Pharmacy,

Aichi Gakuin University, 1-100 Kusumoto-cho, Chikusa-ku,

Nagoya 464-8650, Japan 
684

Journal of Natural Medicines (2019) 73:683-684

Fig. 1 Chemical structures of SPF1, SPF2, and bexarotene

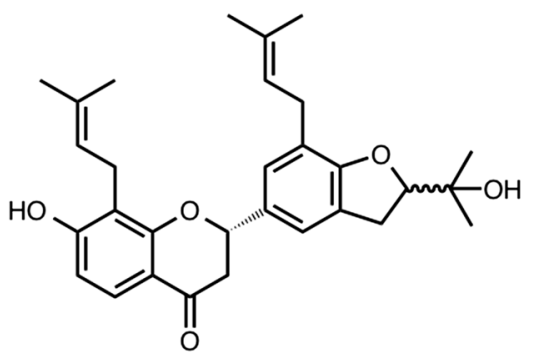

2-[\{3-hydroxy-2', 2-dimethyl-8-(3-methyl2-butenyl)\}chroman-6-yl]-7-hydroxy-8-(3methyl-2-butenyl)-chroman-4-one (SP F1)

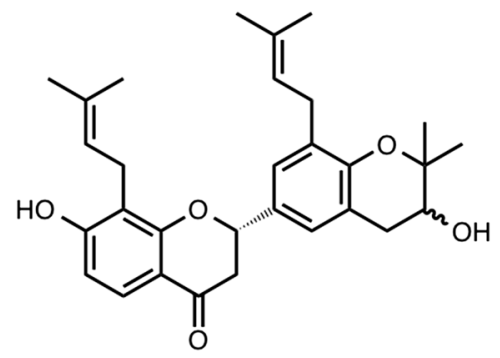

2-[\{2-(1-hydroxy-1-methylethyl)-7-(3-methyl2-butenyl)-2', 3-dihydrobenzofuran\}-5-yl]-7hydroxy-8-(3-methyl-2-butenyl)chroman-4-one (SPF)

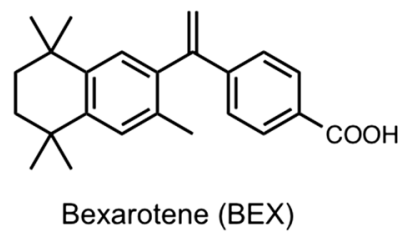

Publisher's Note Springer Nature remains neutral with regard to jurisdictional claims in published maps and institutional affiliations.

Springer 